\title{
DOS NUEVOS TAXA DEL GENERO MIMOSA (LEGUMINOSAE: MIMOSOIDEAE) PARA EL NORTE DE MEXICO
}

\author{
Jose A. Villatreal Q. \\ Universidad Autónoma Agraria "Antonio Narro" \\ Buenavista, Saltillo 25315, Coahuila, México.
}

\begin{abstract}
RESUMEN
Se describen dos taxa nuevos de Mimosa del Desierto Chihuahuense, $M$. setuliseta Villarreal de la porción sur y $M$. emoryana Benth. var. canescens Villarreal de la porción este. Se comparan sus características distintivas, se ilustra su distribución y se presenta una clave para diferenciar estos taxa de otros estrechamente relacionados.
\end{abstract}

\begin{abstract}
Two new taxa of Mimosa are described from the Chihuahuan Desert region, $M$. setuliseta Villarreal from the southern portion and $M$. emoryana Benth. var. canescens Villarreal from the eastern portion. Their known range of distribution is mapped and a key is provided to differentiate the new taxa from closely related ones.
\end{abstract}

El grupo de especies arbustivas de Mimosa que presentan frutos con valvas setosas y que se distribuyen en el noreste de México está formado por: M. emoryana Benth., con distribución en el área del Desierto Chihuahuense y $M$. monancistra Benth. con amplia repartición en el centro y noreste de México.

La revisión de ejemplares de herbario de estas especies con el propósito de aclarar la identidad de muestras de Mimosa con frutos densamente setosos y hojas grandes, nos llevó a la conclusión de distinguir una nueva especie, además de reconocer una variedad nueva de Mimosa emoryana. El presente descubrimiento es parte del trabajo de colecta llevado a cabo en relación con el estudio de la flora del estado de Coahuila realizado por personal del herbario ANSM.

\section{Mimosa setuliseta Villarreal sp. nov.}

Frutex 1.2-1.6 mm altus, pilis sericeis breviter strigosus, aculeis infrastipularibus armatus; foliorum petiolus $10-20 \mathrm{~mm}$ longus, rachis $30-50 \mathrm{~mm}$ longa, pinnae 3-5-jugae et pinnarum foliola 3-5-juga. Flores eos $M$. emoryanae simulantes, calyce infundibuliformi 1.5$2 \mathrm{~mm}$ et corolla campanulata $2-3 \mathrm{~mm}$ longa. Craspedium $40-50 \times 8-11 \mathrm{~mm}$, setis setosis dense obsitum, margine saepissime haud aculeatum. 
Arbusto de 1.2-1.6 m de alto. Ramas jóvenes alargadas, estriadas, de color cafépúrpura, con pubescencia cortamente sericea; aguijones infraestipulares, anchos en la base, recurvados, de color café y pubescentes. Estipulas subuladas $1.5 \mathrm{~mm}$ de largo. Hojas alternas, peciolos $1.5-2.4 \mathrm{~cm}$ de largo, dorsoventralmente comprimidos con un canal central en la cara ventral y una costilla central en la cara dorsal que se continúan en el raquis; pubescencia cortamente serícea densa; raquis $3-5 \mathrm{~cm}$ de largo, con algunos aguijones dorsales, pinnas 3-4(-5) pares, subopuestas, $1.8-3 \mathrm{~cm}$ de largo, con estipelas de $0.5 \mathrm{~mm}$ de largo, foliolos 3-4(-5) pares, elipticos $5-9 \mathrm{~mm}$ largo y 2-3 mm de ancho, oblicuos, de color verde claro a glaucoides, más pálidos en el envés, estrigosos en ambas caras. Inflorescencias simples, axilares y solitarias, pedúnculos $2-3.5 \mathrm{~cm}$ de largo, cabezuelas ovoides a cortamente cilindricas $0.6-1.2 \mathrm{~cm}$ de largo. Flores rosa-rojizas, cortamente seríceas, cáliz infundibuliforme $1.5-2 \mathrm{~mm}$ de largo, lóbulos muy cortos, corola estrechamente campanulada $2-3 \mathrm{~mm}$ de largo, lóbulos de la mitad del largo del tubo, estambres 10, exertos, filamentos $11-12 \mathrm{~mm}$ de largo. Fruto una legumbre de $4-5 \mathrm{~cm}$ de largo y 0.8-1.1 cm de ancho, de 3-7(-8) artículos, normalmente sin aguijones marginales y cubiertos por setas densamente setosas.

TIPO: México. DURANGO. Mpio. de Lerdo, Sierra del Rosario, $40 \mathrm{~km}$ al SW de Cd. Lerdo, $25^{\circ} 25^{\prime} \mathrm{N}, 103^{\circ} 45^{\prime} \mathrm{W}$, alt. $1800 \mathrm{~m}$, matorral de Agave lechuguilla, Acacia crassifulia, A. constricta, Fouquieria, Bursera, Viguiera, Opuntia, 9.XI.1990, J. A. Villarreal 5790 y M. A. Carranza. (Holotipo: MEXU; Isotipos ANSM, ENCB, NY, TEX).

Material adicional examinado: DURANGO. ca 14 air miles WSW of Torreón, 2.5 miles $W$ of Hwy 40 towards microondas est. Sapioris, in $\mathrm{S}$ side of limestone canyon along road, $25^{\circ} 18^{\prime} \mathrm{N}, 103^{\circ} 43^{\prime} \mathrm{W}$, alt. $1800 \mathrm{~m}, 14 . \mathrm{VIII.1973,} \mathrm{J.} \mathrm{Henrickson} 12378$ (TEX); Cerro San Ignacio, mpio. Gómez Palacio, 26.X.1986, E. Blanco 212 (ANSM). TAMAULIPAS. 4 $\mathrm{km}$ al $\mathrm{W}$ del ejido el Salitrillo, mpio. de Tula, alt. $1400 \mathrm{~m}, 3 . \mathrm{VII.1985,} \mathrm{P.} \mathrm{Hiriart} \mathrm{814,} \mathrm{V.}$ Juárez y R. Molczadzki (MEXU). SAN LUIS POTOSI. Paso del Aguila, mpio. de San Nicolás Tolentino, alt. 1600 m, 13.VI.1986, F. Gomez-Lorence 749 (CHAPA); Sta. Catarina, km 75, carr. San Luis Potosi-Rioverde, alt. $1350 \mathrm{~m}$, terrenos aluviales, 3.IX.1965, Rzedowski 6444 (ENCB); 28 km al WNW de la Cd. del Maiz, carr. a San Luis Potosí, alt. $1050 \mathrm{~m}$, terrenos aluviales, 24.VI.1959, Rzedowski 10819 (ENCB).

Mimosa setuliseta parece tener su distribución restringida a las montañas de rocas calizas del sur del Desierto Chihuahuense, en altitudes entre los 1100-1800 m. La vegetación es dominada por matorrales desérticos con abundancia de lechuguilla (Agave lechuguilla), alerón (Acacia crassifolia), guajillo (Acacia berlandieri) y ocotillo (Fouquieria splendens). La distribución comprende una localidad en el NE de Durango y SW de Coahuila, la otra es el área donde confluyen los estados de Nuevo León, Tamaulipas y San Luis Potosi. Johnston (1977) presenta este mismo patrón de distribución disyunta para Acacia crassifolia y Bonetiella anomala, endémicas de estas regiones.

Las rasgos más sobresalientes de esta nueva especie se comparan en el cuadro 1, donde se observa que sus características vegetativas se asemejan a las de $M$. monancistra. Se diferencia por las hojas de mayores dimensiones con pubescencia cortamente serícea y de color verde glauco. Las flores son de dimensiones ligeramente mayores (Fig. 1a) con un cáliz infundibuliforme de lóbulos cortos y agudos, corola campanulada semejante 
a la de $M$. emoryana. El fruto presenta setas, las cuales a su vez tienen prominencias setosas y ápice uncinulado (Fig. 1b), y usualmente carece de aguijones marginales, a diferencia de los frutos de $M$. emoryana que presentan aguijones marginales y setas vilosas y uncinuladas.

El carácter del fruto doblemente setoso (con setas setosas) es al que hace referencia el epíteto específico setuliseta, empleado como nombre descriptivo de la especie.

Cuadro 1. Comparación de caracteres en tres especies relacionadas de Mimosa.

\begin{tabular}{|c|c|c|c|}
\hline Caracter & $\begin{array}{l}\text { Mimosa } \\
\text { monancistra }\end{array}$ & $\begin{array}{l}\text { Mimosa } \\
\text { emoryana }\end{array}$ & $\begin{array}{l}\text { Mimosa } \\
\text { setuliseta }\end{array}$ \\
\hline tallo & zigzagueante & casi recto & casi recto \\
\hline $\begin{array}{l}\text { ramillas } \\
\text { (color) }\end{array}$ & $\begin{array}{l}\text { verde-amari- } \\
\text { llentas }\end{array}$ & $\begin{array}{l}\text { café-amari- } \\
\text { llentas }\end{array}$ & purpúreas \\
\hline pubescencia & $\begin{array}{l}\text { cortamente } \\
\text { sericea }\end{array}$ & sericea & $\begin{array}{l}\text { cortamente } \\
\text { sericea }\end{array}$ \\
\hline aguijones & infraestipulares & internodales & infraestipulares \\
\hline $\begin{array}{l}\text { pecíolo } \\
\text { (tamaño) }\end{array}$ & $3-9 \mathrm{~mm}$ & $6-12 \mathrm{~mm}$ & $15-20 \mathrm{~mm}$ \\
\hline $\begin{array}{l}\text { raquis } \\
\text { (tamaño) }\end{array}$ & $18-20 \mathrm{~mm}$ & $0-15 \mathrm{~mm}$ & $30-50 \mathrm{~mm}$ \\
\hline pinnas & (2)3-4 pares & 1-3(-4)pares & $3-4(-5)$ pares \\
\hline folíolos & 4-5 pares & $3-4(-5)$ pares & $3-4(-5)$ pares \\
\hline pedúnculos & $10-12 \mathrm{~mm}$ & $15-20 \mathrm{~mm}$ & $20-30 \mathrm{~mm}$ \\
\hline $\begin{array}{l}\text { cáliz } \\
\text { (tamaño) }\end{array}$ & $1.0 \mathrm{~mm}$ & $0.8-1.0 \mathrm{~mm}$ & $1.5 \mathrm{~mm}$ \\
\hline $\begin{array}{l}\text { corola } \\
\text { (tamaño) }\end{array}$ & $1.5 \mathrm{~mm}$ & $2.0-2.6 \mathrm{~mm}$ & $2.5-3.0 \mathrm{~mm}$ \\
\hline fruto & $\begin{array}{l}\text { hasta } 7-8 \\
\text { segmentos }\end{array}$ & $\begin{array}{l}\text { hasta } 5-6 \\
\text { segmentos }\end{array}$ & $\begin{array}{l}\text { hasta } 7-8 \\
\text { segmentos }\end{array}$ \\
\hline $\begin{array}{l}\text { setas del } \\
\text { fruto }\end{array}$ & $\begin{array}{l}\text { glabras, ápice } \\
\text { uncinulado }\end{array}$ & $\begin{array}{l}\text { vilosas, porción } \\
\text { apical uncinulada }\end{array}$ & setosas \\
\hline
\end{tabular}



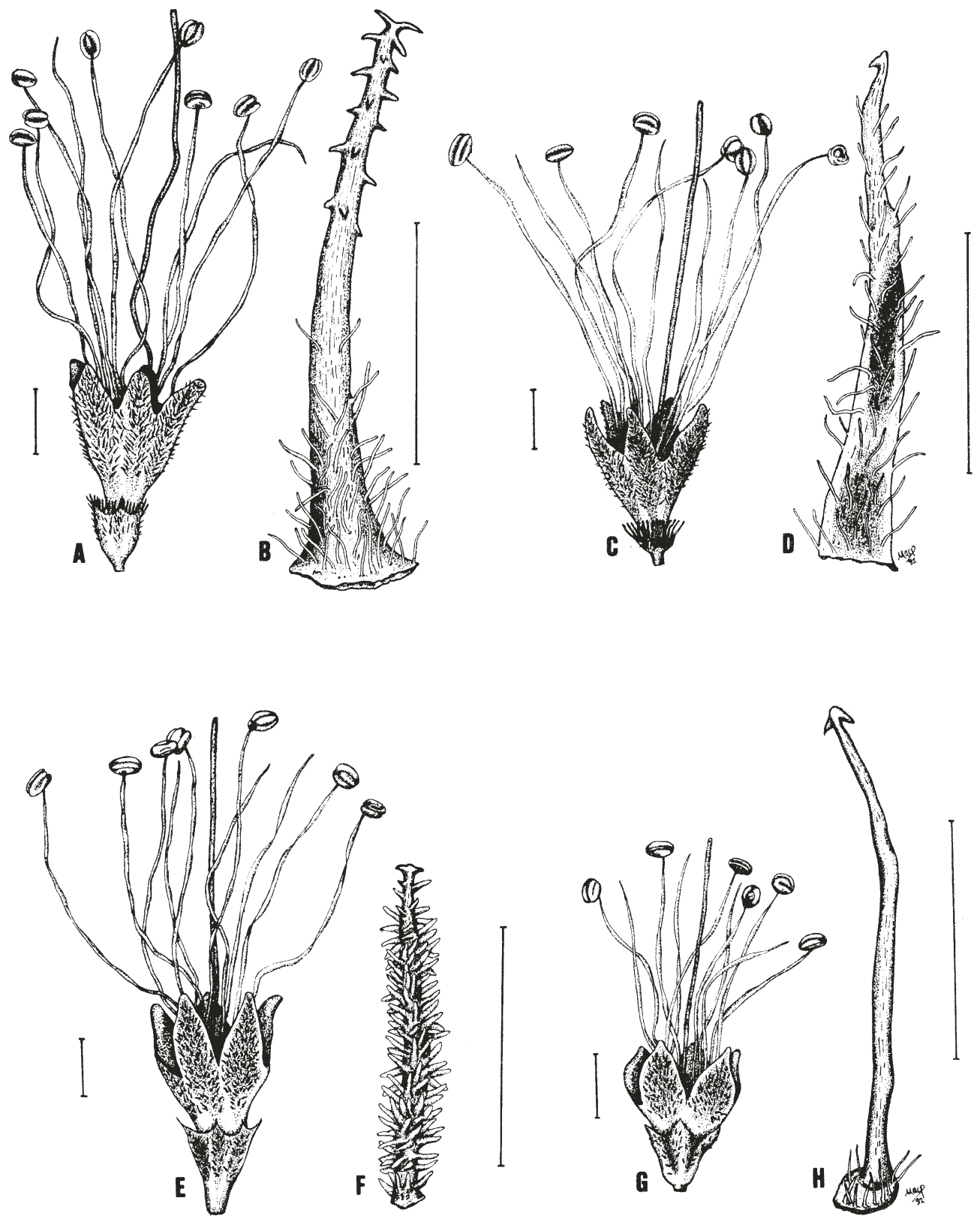

Fig. 1. Flores y setas del fruto de Mimosa emoryana var. emoryana (A y B); $M$. emoryana var. canescens $(C$ y $D) ; M$. setuliseta $(E$ y $F)$ y $M$. monancistra $(G$ y $H)$. Todas las barras de referencia corresponden a $1 \mathrm{~mm}$. 


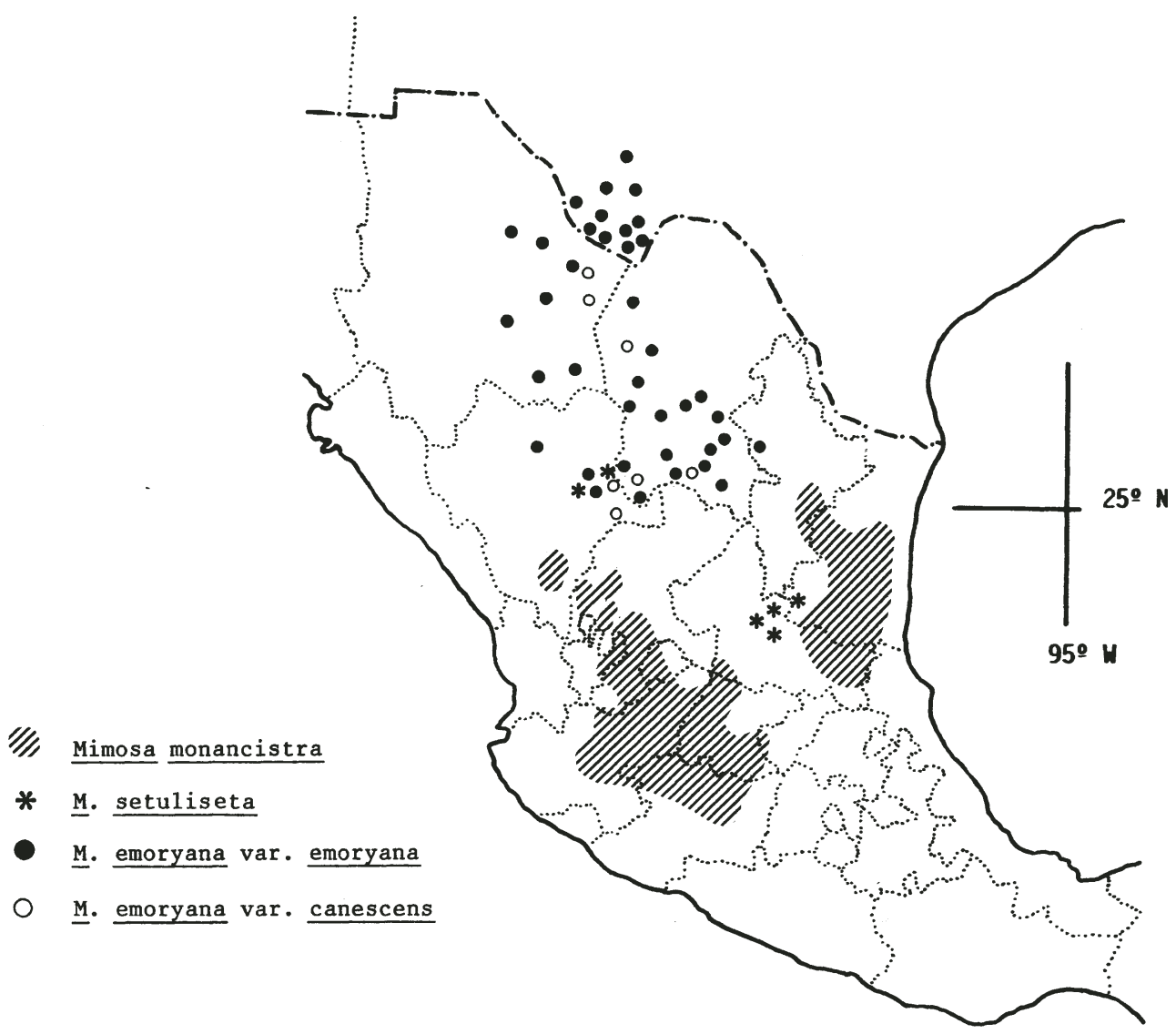

Fig. 2. Distribución conocida de Mimosa monancistra, M. setuliseta, M. emoryana var. emoryana y var. canescens en México y sur de Texas.

De acuerdo con Robinson (1898), Mimosa emoryana fue descrita por G. Bentham en 1875 del ejemplar colectado por Bigelow No. 312 en Cibolo, cerca del Rio Grande, Texas, en 1852. La localidad es algo confusa, ya que Cibolo se localiza en un área cercana a San Antonio, retirado del Rio Grande. Todos los ejemplares examinados de Texas fueron colectados en el sur de los condados de Brewster y Presidio, en el área del Trans-Pecos en las proximidades de los límites con México, lo que nos permite pensar que la localidad tipo debe estar en esta área próxima al Rio Grande.

La distribución de $M$. emoryana se extiende desde el área antes mencionada de Texas hasta el sur de Coahuila y noreste de Durango (Fig. 2). En el centro del área de distribución de la especie se encuentran ejemplares con una serie de caracteres diferentes del resto de la población y que se describen a continuación como una variedad nueva. 
M. emoryana Benth. var. canescens Villarreal var. nov.

A var. emoryana pube densissime sericea, floribus minoribus, calyce $0.5-0.8 \mathrm{~mm}$ longo conspicue ciliato, corolla $1.5-1.8 \mathrm{~mm}$ longa, craspedii margine incrassata, necnon valvularum setis complanatis villosis sed apice tantum uncinulatis diversa.

M. emoryana Benth., con hojas de 1(-2) pares de pinnas densamente seríceas. Flores pequeñas, cáliz de 0.5-0.8 mm de largo, borde ciliado, corola de 1.5-1.8 mm de largo. Fruto con el borde engrosado, setas de las valvas aplanadas, vilosas y con el ápice uncinulado.

TIPO. México. Durango. Mpio. Cuencamé. 4 mi. N of Pedriceña, turnoff along Hwy $40,50 \mathrm{ft} \mathrm{N}$ of microondas est. Sierra Lorenzo in igneous rocky slopes, $29^{\circ} 09^{\prime} \mathrm{N}, 103^{\circ} 48^{\prime} \mathrm{W}$, alt. $4500 \mathrm{ft}$, common shrub to $1 \mathrm{ft}$ high, $3 \mathrm{ft}$ diameter, fls. rose-pink; with Agave lechuguilla, Opuntia, Acacia spp., Larrea, Jatropha, Fouquieria, etc., 13.VIII.1973, J. Henrickson \& T. Wendt 12312 (HOLOTIPO: TEX).

Material adicional examinado: COAHUILA. Mpio. Fco. Madero, $135 \mathrm{mi} \mathrm{W}$ of Saltillo on Hwy 40 edge of Bolson of Mapimi, 23.VIII.1984, CDRI 1062 (TEX); Gral. Cepeda, Sierra Narigua $25^{\circ} 36^{\prime} \mathrm{N}, 101^{\circ} 35^{\prime} \mathrm{W}$, alt. 1200 m, 17.X.1989, D. Castillo 1070, 1115 (ANSM). CHIHUAHUA. Mpio. Aldama. Just S and SE of Mina La Nueva Esperanza on the eastern slope, Sierra El Morrión, gravelly calcareous loam, $28^{\circ} 55^{\circ} \mathrm{N}, 105^{\circ} 31^{\prime} \mathrm{W}$, alt. $1400-1700$ m, 10.VII.1972, F. Chiang. T. Wendt \& M. C. Johnston 8392 (MEXU,TEX). mpio. de Ojinaga, 6 mi NE of Cuchillo Parado, open Chihuahuan Desert in rocky clay area. $29^{\circ} 30^{\prime} \mathrm{N}, 104^{\circ}$ 45'W, alt. $3400 \mathrm{ft}, 17 . \mathrm{IX} .1971$. J. Henrickson 6788 (TEX); $5 \mathrm{~km}$ airline W of Alamo Chapo. on the Ojinaga-La Perla Hwy. $29^{\circ} 19^{\prime} \mathrm{N}, 104^{\circ} 23^{\prime} \mathrm{W}$, limestone-calcareous soil, alt. $925 \mathrm{~m}$, 3.V.1973, M. C. Johnston, T. Wendt \& F. Chiang 10790 (MEXU,TEX). DURANGO. Mpio. Cuencamé, on rocky limestone slope, 2 mi NW of Pedriceña, route 31, 23.VII.1958, D. S. Correll \& I. M. Johnston 20027 (TEX-LL); CNIZA Cuencamé, 22.VIII.1978, L. Luna O. s/n. (ANSM); $8 \mathrm{mi} \mathrm{N}$ of Cuencamé, 27.VI.1977, J. D. Dwyer 14250 (MEXU,MO).

La variedad canescens se distribuye en la porción mexicana del Desierto Chihuahuense en áreas de matorral de Agave lechuguilla, Fouquieria splendens y Opuntia rastrera. Esta variedad presenta una serie de caracteres adaptativos a condiciones de fuerte aridez; entre los más sobresalientes destacan las estructuras florales más pequeñas y hojas con pubescencia densa, como formas de protección contra el efecto deshidratante del ambiente.

El nombre de la variedad hace referencia a la pubescencia densa que cubre a hojas y ramas jóvenes de la planta, con apariencia grisácea, diferente de la variedad típica.

Los caracteres diferenciales para separar los taxa antes mencionados se presentan en la siguiente clave:

1. Tallos zigzagueantes con ramas jóvenes verde-amarillentas; flores $2.5 \mathrm{~mm}$ de largo; frutos con setas uncinuladas glabras. Jal., Mich., Ags., Gto., Qro., S.L.P., Zac., Dgo., N.L. y Tamps. M. monancistra 
1. Tallos casi rectos con ramas jovenes amarillentas o purpúreas; flores $3.0-3.5 \mathrm{~mm}$ de largo; fruto con setas uncinuladas pero no glabras.

2. Frutos densamente setosos, las setas a su vez setosas, con el ápice ligeramente uncinulado; hojas $45-70 \mathrm{~mm}$ de largo, 3-5 pares de pinnas; aguijones infraestipulares. NE de Dgo., SW de Tamps. y N de S. L. P. ........ setuliseta

2. Frutos con setas vilosas y uncinuladas; hojas $0.6-2.8 \mathrm{~cm}$ de largo, 1-3 pares de pinnas; aguijones internodales. Tex., Chih., Dgo., Coah. y N.L. (M.emoryana)

3. Cáliz 0.5-0.8 mm de largo, conspicuamente ciliado; fruto con setas aplanadas, vilosas, con el ápice uncinulado; hojas con pubescencia densamente serícea.

M. emoryana var. canescens

3. Cáliz $0.8-1.0 \mathrm{~mm}$ de largo, ciliado; fruto con setas cilíndricas, vilosas en la base y uncinuladas en la porción apical; hojas con pubescencia sericea.

M. emoryana var. emoryana

\section{AGRADECIMIENTOS}

Agradezco al Dr. R. Barneby su colaboración en la revisión del manuscrito y las traducciones de las diagnosis al latín. A Miguel A. Carranza la elaboración de las ilustraciones y su compañla en las colectas de campo. A los encargados de los herbarios ENCB, MEXU y TEX por el préstamo de ejemplares.

\section{LITERATURA CITADA}

Isely, D. 1973. Leguminosae of the United States: I. Subfamily Mimosoideae. Mem. New York Bot. Gard. 25(1): 99-109.

Johnston, M. C. 1977. Brief resume of botanical, including vegetational features of the Chihuahuan Desert Region with special emphasis on their uniqueness. In: Wauer, R. H. \& D. H. Riskind (ed.) U.S. National Park Service Trans. Proc. Series 3: 335-359.

Robinson, B. L. 1898. Revision of the North American species of Mimosa. Proc. Amer. Acad. Sc. (Contr. Gray Herb. no 13.) 33: 305-334.

Standley, P. C. 1922. Trees and shrubs of Mexico. (Mimosa). Contr. U.S. Nat. Herb. 23(2): 355-366. 